\title{
Railway Vehicle Pneumatic Rubber Suspension Modelling and Analysis
}

\begin{abstract}
MARIUS ADRIAN SPIROIU*
Politehnica University of Bucharest, Faculty of Transports, 313 Splaiul Independentei, 060042, Bucharest, Romania

The pneumatic elastic elements are widely used in the secondary suspension of modern railway vehicles due to their important advantages compared to the conventional ones. In the present paper, the modelling and analysis of the pneumatic suspension is approached, taking into account the main elements, pneumatic enclosures and rubber elements. The analysis performed investigates the influence of relevant parameters on suspension stiffness and on its dynamic response.
\end{abstract}

Keywords: railway vehicle, pneumatic suspension, rubber spring

Nowadays, the main tendencies in rail transport are to increase the transport capacity and the traveling speed of the vehicles, providing at the same time a complete safety of traffic and a good ride quality. In both of these latter issues, the suspension system performances are essential. A railway vehicle in motion is subject to vibrations in both vertical and horizontal plane, the sources of these vibrations being track irregularities, rail discontinuities (at the joints, switches and crossings), wheel defects (such as eccentricity, wheel flats), the rigid fastening of the wheels to the axle, etc. [1]. Under these circumstances, the railway vehicle suspension has to isolate the car body from the vibrations determined by the perturbations mentioned above. The suspension system has to ensure a stable dynamic behaviour of the vehicle in straight line and in curves and to reduce the vehicle-track mutual forces, for reasons of traffic safety, passenger comfort and protection of both wheelset and track [1, 2].

Although there are some railway vehicles that have one or more than two suspension stages, in most cases the suspension consists of two stages: primary suspension, between the wheelsets and the bogie frame and secondary suspension, between the bogie frame and the bolster or car body. The primary suspension is stiffer, in order to ensure vehicle stability and guidance, while the secondary suspension is softer, in order to ensure passenger comfort. A suspension stage consists of elastic elements (springs), dampers and connection elements. The first suspension systems used metallic elastic elements - such as coil springs, leaf springs and torsion springs. Coil springs are still the most commonly used elastic elements, especially because of their simplicity. How ever, along with the technological progress, some other types of elastic elements (e.g. rubber springs, air springs) have emerged and have been introduced instead of conventional steel springs. Pneumatic suspensions incorporating air springs are widely used in modern passenger cars due to their superior performance compared to traditional suspension systems. The pneumatic suspension is used exclusively in the secondary suspension of metro trains and is also practically generalized in modern passenger vehicles, including high-speed trains. In the case of the French highspeed trains, for example, if the first generation of French high-speed trains (TGV Paris South East, 1981) was fitted with coil springs, starting with the second generation (TGV Atlantique, 1989), the secondary suspension coil springs have been replaced with air springs [3].
Structure and operation of the pneumatic suspension

Air springs are used in the secondary suspension of railway vehicles and are using their compressed air supply system. In figure 1 is shown a simplified structure of a pneumatic suspension corresponding to a quarter of a vehicle $[3,4]$.

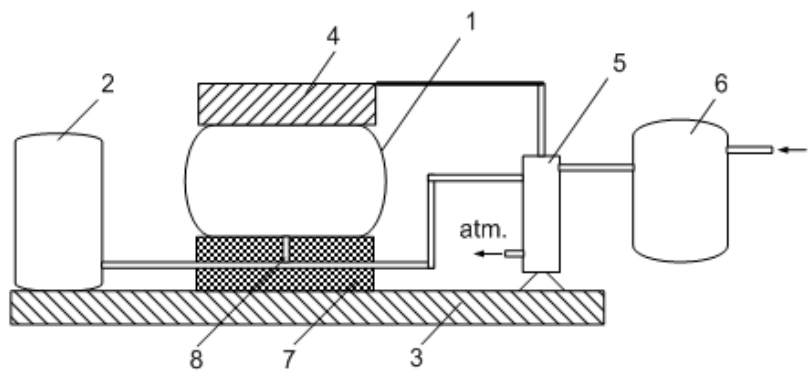

Fig. 1 Pneumatic suspension system [3, 4]

The pneumatic suspension consists of an air spring 1 (rubber airbag) located between the bogie 3 and the car body 4 , connected to an auxiliary reservoir 2 . The central pneumatic suspension includes usually a rubber spring 7 arranged in series with the air spring, in order to provide extra flexibility and to act as a safety device in the case of air spring deflation.

One of the main features of the pneumatic suspension is the ability to maintain a constant height of the car body, regardless of vehicle static load. This is achieved through the levelling valve 5 , which controls the air flow between the air spring 1, the main reservoir 6 and the atmosphere. Thus, it can either increase the air spring internal pressure by connecting it to the main reservoir (if the static load increases) or to decrease the air spring internal pressure by connecting it to the atmosphere (if the static load decreases). In this way itis obtained a stiffness progressive with the static load. Adjusting the stiffness with the load allows also maintaining comfortable vibration frequencies for the entire range of static load.

As the air spring axial stiffness is inversely proportional to the volume, the auxiliary reservoir is necessary in order to increase the total volume of air, thus to reduce the spring stiffness. The air flows from the air bag to the auxiliary reservoir during the spring compression (loading) then the flow is reversed during the rebound (unloading). By placing a calibrated orifice between the air bag and the auxiliary reservoir, it is created a flow resistance to air circulation, thus a damping effectis obtained. The damping magnitude is more important as the orifice size is smaller. 
The pneumatic suspension structure and specific mode of operation causes the frequency of vibration to influence the suspension characteristics. In the case of high frequencies the air no longer flows between the air bag and the auxiliary reservoir, so only the volume of the air bag contributes to provide axial stiffness. Also, the air spring deformation velocity - given by frequency of oscillation influences the thermodynamic transformation of air: for frequencies below $0.1 \mathrm{~Hz}$ an isothermal transformation takes place, while for frequencies higher than $3 \mathrm{~Hz}$ an adiabatic process occurs. Taking into account the usual frequency range of car body vertical vibrations, the transformation of the compressed air within the air suspension is polytropic.

\section{Modelling the pneumatic suspension}

There are many approaches to the analysis and modelling of pneumatic suspensions. In some cases these analyses are based on experimental observations, such as in [5], where is proposed an analytical model of air spring suspension which includes only the air spring, the auxiliary reservoir and the connecting pipe, in [6], where is developed a model of a controlled pneumatic spring or in [7], where two mathematical modelling approaches, a quasi-static one and a dynamic one, are developed. In [8] are reviewed existing models for railway vehicle suspension components and their use for railway vehicle dynamics multi-body simulations, a particular importance being given to the pneumatic suspensions. Analytic models for air springs are proposed in $[3,9,10]$, where are also investigated the effects of air volumes and the connecting pipes dimensions on the suspension performances. The models in the above-mentioned works focus on the assembly air bag - auxiliary reservoir. How ever, in [11] are mentioned, as simple sketches, several possible air spring models, among which one includes also the rubber spring, but no further analysis is performed on this model, being preferred simpler models, such as Kelvin-Voigt and Nishimura.

Under these circumstances, in the present paper is approached a model of the pneumatic suspension which includes the air bag, the auxiliary reservoir and the rubber spring. Modelling the air suspension is based on the expression of its axial stiffness, given by

$$
k_{p}=\frac{d F}{d z}=\frac{d\left(p A_{e f}\right)}{d z}=p \frac{d A_{e f}}{d z}+A_{e f} \frac{d p}{d z},
$$

where $F$ is the vertical load, $z$ - the vertical deflection, $p$ the air pressure within the spring and $A_{e f}$ is the air bag eûective area, i.e. the area of the air spring considered to be the load carrying area. The stiffness in equation (1) has two terms, showing that it depends on the variation of effective area and that of the air pressure. The first term is given by the variation of air spring diameter during its operation. The magnitude of this stiffness is generally not very important, depending also on the air spring type. The air spring stiffness given by the second term can be written, in the conditions of a polytropic transformation, as:

$$
k=\frac{A_{e f}^{2} p n}{V},
$$

where $n$ is the polytropic coefficient and $V$ is the volume of air spring. If $\alpha$ denotes the ratio of air spring and auxiliary reservoir volumes

$$
\alpha=V / V_{\text {aux }}
$$

the stiffness corresponding to the auxiliary reservoir can be written as:

$$
k_{\text {aux }}=\frac{{A_{e f}}^{2} p n}{V_{a u x}}=\alpha k
$$

Therefore, the suspension model for a quarter-car is shown in figure 2, where: $m$ - is the quarter-car body mass; $k_{1} \alpha k_{1} k_{1}$ and $k_{2}$ - air spring and auxiliary reservoir stiffness given by pressure variation, air spring stiffness given by effective area variation, rubber spring stiffness, respectively; $c_{1}$ and $c_{2}$ - damping constants of air spring-auxiliary reservoir ensemble and of rubber spring; $z$ and $z_{0}$-car body and bogie vertical displacements.

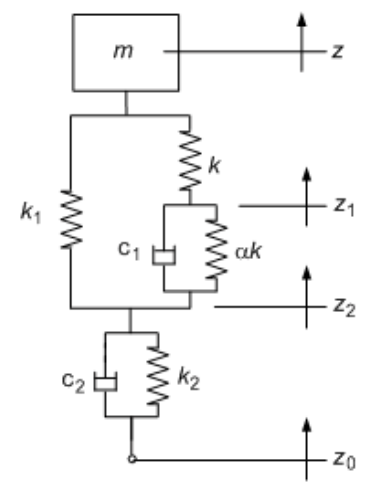

Fig. 2 Pneumatic suspension model

The motion equations of the model in figure 2 can be written as:

$$
\begin{aligned}
& m \ddot{z}+k\left(z-z_{1}\right)+k_{1}\left(z-z_{2}\right)=0 \\
& k\left(z_{1}-z\right)+\alpha k\left(z_{1}-z_{2}\right)+c_{1}\left(\dot{z}_{1}-\dot{z}_{2}\right)=0 \\
& k_{1}\left(z_{2}-z\right)+\alpha k\left(z_{2}-z_{1}\right)+c_{1}\left(\dot{z}_{2}-z_{1}\right)+k_{2}\left(z_{2}-z_{0}\right)+c_{2}\left(\dot{z}_{2}-z_{0}\right)=0 \\
& \text { Since one of the main aims of the paper is to analyze the } \\
& \text { dynamic behaviour of the pneumatic suspension, its } \\
& \text { frequency response factor has to be determined. } \\
& \text { Using the complex notations } \\
& \qquad \bar{z}_{0}=Z_{0} \exp (i \omega t) ; \bar{z}_{1}=Z_{1} \exp \left(i \omega t+\varphi_{1}\right) ; \\
& \qquad \bar{z}_{2}=Z_{2} \exp \left(i \omega t+\varphi_{2}\right) ; \bar{z}=Z \exp \left(i \omega t+\varphi_{3}\right)
\end{aligned}
$$

and substituting in equations (5), after some calculations is obtained the complex transfer function between the car body and bogie vertical displacements.

where:

$$
\bar{H}_{z}(\omega)=\frac{\bar{z}}{\bar{z}_{0}}=\frac{A}{B-C}
$$

$$
\begin{gathered}
A=k\left(k_{2}+\mathrm{i} \omega c_{2}\right)\left[k\left(\alpha k+\mathrm{i} \omega c_{1}\right)+k_{1}\left(k+\alpha k+\mathrm{i} \omega c_{1}\right)\right] \\
B=\left[\left(k+k_{1}-m \omega^{2}\right)\left(k+\alpha k+\mathrm{i} \omega c_{1}\right)-k^{2}\right]\left[k_{1}\left(\alpha k+\mathrm{i} \omega c_{1}\right)+\right. \\
\left.+k\left(\alpha k+k_{1}+k_{2}+\mathrm{i} \omega c_{1}+\mathrm{i} \omega c_{2}\right)\right] \\
C=\left[k k_{1}+\left(\alpha k+\mathrm{i} \omega c_{1}\right)\left(k+k_{1}-m \omega^{2}\right)\right]\left[k_{1}\left(k+\alpha k+\mathrm{i} \omega c_{1}\right)+\right. \\
\left.+k\left(\alpha k+\mathrm{i} \omega c_{1}\right)\right]
\end{gathered}
$$

Defining the natural circular frequency $\omega_{0}$ and the damping ratios $\xi_{1}, \xi_{2}$

$$
\omega_{0}=\sqrt{\frac{k}{m}} ; \zeta_{1}=\frac{c_{1}}{2 \sqrt{k m}} ; \zeta_{2}=\frac{c_{2}}{2 \sqrt{k m}}
$$

and denoting $\lambda=\frac{\omega}{\omega_{0}} ; \beta_{1}=\frac{k_{1}}{k} ; \beta_{2}=\frac{k_{2}}{k}$ the terms $A, B$ and $C$ in equations (7) can be written under the form: 


$$
\begin{aligned}
& A=\left(\beta_{2}+2 j \lambda \zeta_{2}\right)\left[\alpha+\beta_{1}(1+\alpha)+2 j \lambda \zeta_{1}\left(1+\beta_{1}\right)\right] \\
& B=\left[\left(1+\beta_{1}-\lambda^{2}\right)\left(1+\alpha+2 j \lambda \zeta_{1}\right)-1\right]\left[\beta_{1}\left(\alpha+2 j \lambda \zeta_{1}\right)+\alpha+\beta_{1}+\beta_{2}+2 j \lambda\left(\zeta_{1}+\zeta_{2}\right)\right] \\
& C=\left[\beta_{1}+\left(\alpha+2 j \lambda \zeta_{1}\right)\left(1+\beta_{1}-\lambda^{2}\right)\right]\left[\beta_{1}\left(1+\alpha+2 j \lambda \zeta_{1}\right)+\alpha+2 j \lambda \zeta_{1}\right]
\end{aligned}
$$

The moduls of the transfer function is:

where:

$$
H_{z}(\omega)=\left|\bar{H}_{z}(\omega)\right|=\sqrt{\frac{A^{\prime}}{B^{\prime}+C^{\prime}}}
$$

$$
\begin{gathered}
A^{\prime}=\left\{\beta_{2}\left[\alpha+\beta_{1}(1+\alpha)\right]-4 \lambda^{2} \zeta_{1} \zeta_{2}\left(1+\beta_{1}\right)\right\}^{2}+4 \lambda^{2}\left[\left(1+\beta_{1}\right)\left(\beta_{2} \zeta_{1}+\alpha \zeta_{2}\right)+\beta_{1} \zeta_{2}\right]^{2} \\
B^{\prime}=\left\{\left(1+\beta_{1}-\lambda^{2}\right)\left[\alpha+(1+\alpha)\left(\beta_{1}+\beta_{2}\right)-4 \lambda^{2} \zeta_{1} \zeta_{2}\right]-\beta_{2}-\left(1+\beta_{1}\right)\left[a+\beta_{1}(1+\alpha)\right]\right\}^{2} \\
C^{\prime}=4 \lambda^{2}\left\{\zeta_{1}\left[\beta_{2}\left(1+\beta_{1}-\lambda^{2}\right)-\lambda^{2}\left(\beta_{1}+1\right)\right]+\zeta_{2}\left[(1+\alpha)\left(1+\beta_{1}-\lambda^{2}\right)-1\right]\right\}^{2}
\end{gathered}
$$

It can be determined also the equivalent stiffness of the suspension according to the model in figure 2 :

$$
k_{\varepsilon}=k \frac{\beta_{2}\left(\beta_{1}+\frac{\alpha}{1+\alpha}\right)}{\beta_{1}+\beta_{2}+\frac{\alpha}{1+\alpha}} .
$$

\section{Results and discussions}

On the basis of the above presented model, in this section is made an analysis of the influence of pneumatic suspension characteristics on its performances. The model parameters are considered to be $m=9600 \mathrm{~kg}, \mathrm{k}=1.2 .10^{6}$ $\mathrm{N} / \mathrm{m}, \mathrm{a}=0.6, \beta_{1}=0.1, \beta_{2}=4, \xi_{2}=0.2, \xi_{2}=0.3$. These are the nominal values, but during the analysis fr some of the parameters there will be considered value ranges.

In figures 3, 4 and 5 is shown the influence on the equivalent stiffness of the suspension of the stiffness given by auxiliary reservoir volume, of the stiffness caused by the variation of air spring effective area and of the stiffness of the additional rubber spring, respectively. The stiffness mentioned above are given by parameters $\alpha, \beta$ and $\beta_{2}$, respectively, and the suspension stiffness $k_{e}$ is considered as its ratio to air spring stiffness $k$. In figure 3 can be seen that volumes ratio a has an important influence on suspension stiffness. For a given air spring volume, increasing the volume of the auxiliary reservoir (i.e. decreasing a) leads to a significantly reduced suspension stiffness.

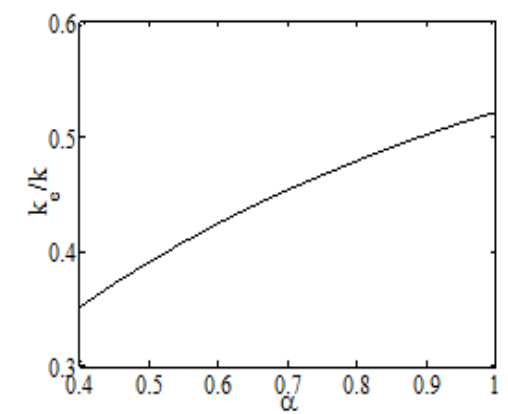

Fig. 3 Influence of parameter $\alpha$ on the equivalent stiffness of the suspension

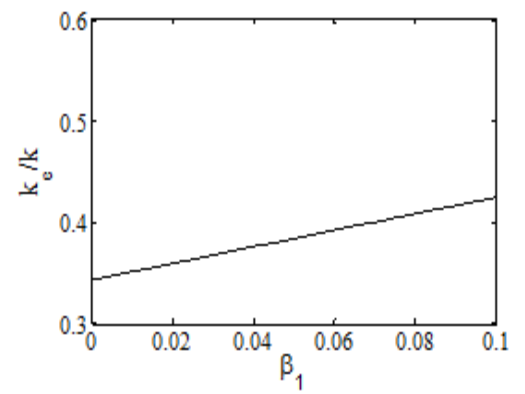

Fig. 4 Influence of parameter $\beta_{1}$ on the equivalent stifness of the suspension
Even if the magnitude of the stiffness given by air spring area variation is not very important, it can be seen in fig. 4 that ithas quite an important influence, its increase resulting in a stiffer pneumatic suspension. On the other hand, the stiffness of the additional rubber spring has a reduced impact on the equivalent suspension stiffness (fig. 5), especially in the range of its usual stiffness values, thus confirming its main purpose of being a safety element.

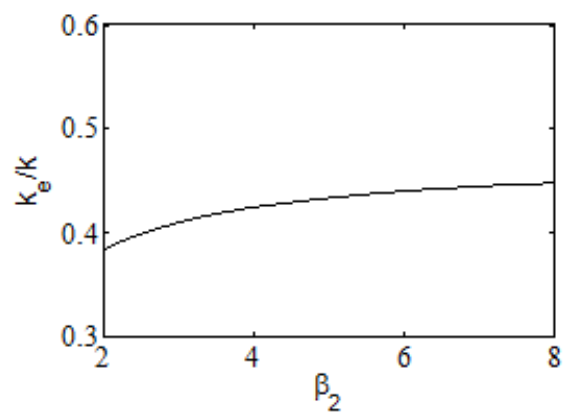

Fig. 5. Influence of parameter $\beta_{2}$ on the equivalent stiffness of the suspension

Concerning the dynamic behaviour of the pneumatic suspension system, the analysis is made based on the frequency response factor given by equation (9). The influence of volumes ratio $\alpha$ is shown in figure 6 . It can be seen that a higher ratio $\alpha$ (a smaller auxiliary reservoir volume (results in a slightly higher natural frequency and in an increased amplification of the vibrations, especially in the resonance area. For better dynamic performances of the suspensions, a lower volumes ratio is then necessary.

In figure 7 is shown the influence, through the values of parameter $\beta_{1}$, of the stiffness given by air spring area

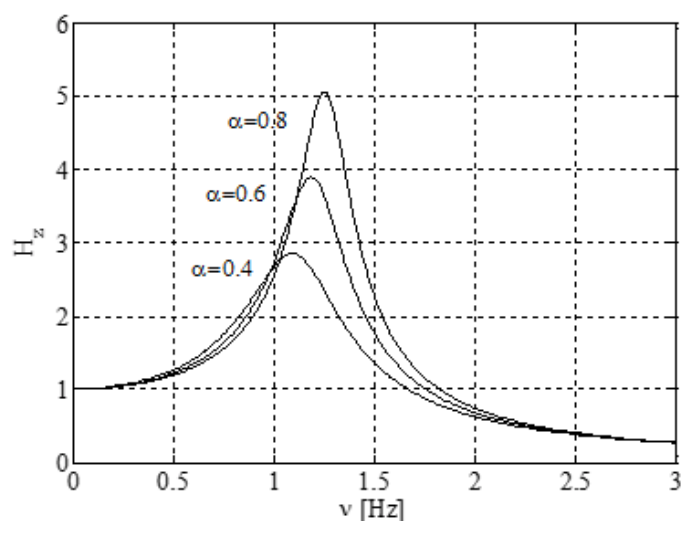

Fig. 6. Influence of parameter $\alpha_{2}$ on the frequency response factor $\mathrm{H}_{z}$ 


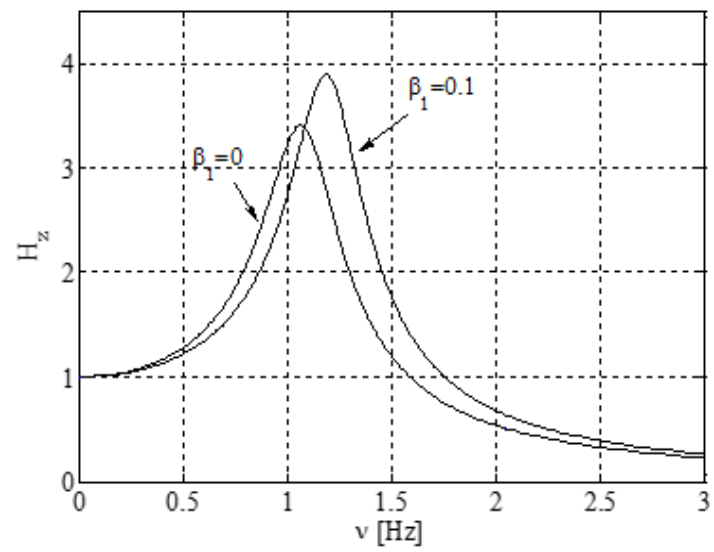

Fig. 7. Influence of parameter $\beta_{1}$ on the frequency response factor $\mathrm{H}_{2}$

variation during operation. It was previously stated that the equivalent stiffness is increased with the increase of $\beta_{1}$, consequently a higher natural frequency is obtained. Also, in this case, a better attenuation is achieved in the domain of low frequencies (below the natural frequency), but a higher amplification in the area of the natural frequency and over it.

In figure 8 is presented the influence of the additional rubber spring stiffness, for which two limit values are considered. It can be seen that the increase of this stiffness ( $\beta$ from 2 to 8 ) leads to a slightly higher natural frequency. $A$ stiffer rubber spring provides a better reduction of vibrations for frequencies lower than the natural one. For higher frequencies, a softer spring is more suitable. It should be mentioned, however, that the effect of this spring on the dynamic performances is not important in comparison with the influences of the other factors investigated above.

\section{Conclusions}

Pneumatic suspensions incorporating air springs are widely used in modern passenger cars due to their superior performance compared to traditional suspension systems. It can be mentioned the ability of maintaining a constant height of the car body by providing a stiffness progressive with the static load, providing also comfortable vibration frequencies for the entire range of static load.

In the present paper, the modelling and analysis of the pneumatic suspension was approached, taking into account the main elements, pneumatic enclosures and rubber components. The equivalent stiffness of the suspension was obtained and the frequency response of the car body vertical displacement was determined. The

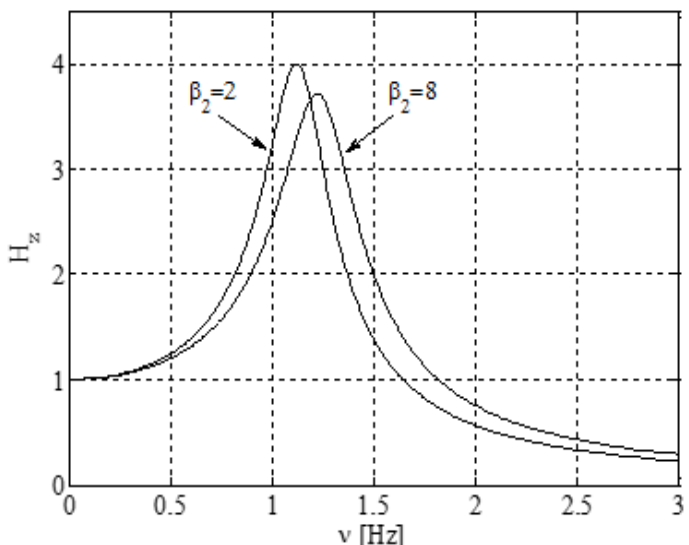

Fig. 8. Influence of parameter $\beta_{2}$ on the frequency response factor $\mathrm{H}_{2}$

analysis performed allowed identifying the influence of relevant parameters on suspension stiffness and on suspension dynamic performances. In all respects, the most important parameter was proven to be the ratio of air spring and auxiliary reservoir volumes $\alpha$. A low value of this ratio allows reducing suspension stiffness and has a positive impact on the suspension performances related to vibration attenuation. The influences of the stiffness caused by the variation of air spring effective area and of the additional rubber spring are less important, yet worthy of consideration, especially the first one.

\section{References}

1. SEBESAN, I., SPIROIU, M.A., ARSENE, S., POPA, G., DINU, G., Mat. Plast., 52, no. 4, 2015, p. 560

2. SEBESAN, I., ZAHARIA, N.L., SPIROIU, M.A., FAINUS, L., Mat. Plast., 52, no. 1, 2015, p. 93

3. SPIROIU, M., Tehnica marilor viteze in transportul feroviar, Ed. Matrix Rom, Bucuresti, 2012

4. IWNICKI, S., Handbook of Railway Vehicle Dynamics, CRC Press, Taylor \& Francis Group, Boca Raton, 2006

5. NIETO, A.J., MORALES, A.L., GONZALES, A., CHICHARRO, J.M., PINTADO, P., J. Sound Vib., 313, 2008, p. 290

6. PALOMARES, E., NIETO, A.J ., MORALES, A.L., CHICHARRO, J.M., PINTADO, P., Mechatronics, 45, 2017, p. 37

7. FACCHINETTI, A., MAZZOLA, L., ALFI, S., BRUNI, S., Veh. Syst. Dyn., 48, Supplement 1, 2010, p. 429

8. BRUNI, S., VINOLAS, J., BERG, M., POLACH, O., STICHEL, S., Veh. Syst. Dyn., 49, Iss. 7, 2011, p. 1021

9. LEE, S.J., Int. J. Automot. Technol., 11, No. 4, 2010, p. 471

10. SAYYAADI, H., SHOKOUHI, N., Iranian J. Sci. Technol., Transaction B: Engineering, 34, no. B5, 2010, p. 499

Manuscript received: 29.11.2017 